\title{
La dialéctica uno-mucho y su conocimiento, en Aristóteles
}

\author{
Héctor Velázquez Fernández \\ Universidad Panamericana
}

Aristotle wonders whether the One is prior to the Many, or conversely. Indeed, the Many seems to bean aggregate of several Ones; but, in order to understand the One, it has to be compared with Many others. This article evaluates Aristotle's approach to this problem, according to the reading and solution given by Aquinas in his Commentary on Metaphysics.

Si el ente es el primum cognitum ${ }^{1}$, lo que primeramente adviene a nuestro intelecto, esto es, si lo primero que consideramos es que las realidades son, a su vez ello no sería posible sino en función del contraste: cuando sé que una cosa no es otra, es cuando entiendo que una cosa es esa cosa. No puedo oponer un ser a otro ser cuando no considero ambos como existentes, y después como diferentes. Es conocida la referencia aristotélica a la necesidad del contraste en el conocimiento ${ }^{2}$.

${ }^{1}$ TOMÁs DE AQUino, Summa Theologiae, 1-2. q.94, a.2, c.; De Veritate, q.1, a.1.: "illud quod primo intellectus concipit quasi notissimum et in quo omnes çonceptiones resolvit est ens".

2 De hecho, como se colige de la exposición aristotélica desarrollada en varios pasajes, acerca del medio en el conocimiento, si viéramos sólo el blanco, nunca podriamos captar los colores, pues a partir del contraste es que conocemos que el negro no es el blanco y que ambos son diferentes: ARISTÓTELES, De anima, II, 418a26-41b4; TOMÁS DE AQUINO, In de anima, lect. 14, nn. 399-426, lect.15, nn. $427-438$ (Marietti). 
Sin embargo, parece haber una cierta circularidad en esta exposición: ciertamente, si dos realidades ya son -en principio pueden ser diferentes-, de lo contrario, ¿por qué digo que son "dos"? ¿Y acaso no es gracias a la diferencia entre ambos, que me doy cuenta de que son? Así, pues, ¿qué conocemos primero, el que algo es o que es diferente a otro? Si considero lo diferente es porque antes lo he concebido como ya existente.

Esta pregunta es abordada por Aristóteles al estudiar la relación entre unidad y pluralidad. La cuestión es dilucidar si alguna de estas realidades guarda prioridad ontológica sobre la otra o si sólo es una preeminencia gnoseológica que depende de nuestras condiciones humanas de conocimiento. Nos acercaremos someramente al problema, para mostrar los diferentes sentidos de la noción de unidad necesarios para poder dilucidar la aparente aporía enunciada. La problemática está contextualizada en el tema de la contrariedad real, la cual funda la diferencia ontológica. No abordaremos el análisis a partir del cual Aristóteles establece el sentido de uno según el cual se justifica la oposición con lo mucho. Más bien, partiendo de las conclusiones sobre la ratio de indivisión, destacaremos los elementos necesarios para mostrar la dialéctica uno-mucho y su conocimiento problemático, pues parece suponer la circularidad ya mencionada.

El problema parece estar planteado en los siguientes términos: aparentemente me doy cuenta de que algo es unidad porque lo confronto con la pluralidad que le circunda; y al contrario, la pluralidad no es otra cosa que un conjunto de unidades; iqué noción tiene prioridad ontológica o gnoseológica sobre la otra?

Después de establecer a lo largo del pasaje 1052al5-b18 del libro $\mathrm{X}$ de la Metafisica, como esencial al uno -estudiado en sí mismo ${ }^{3}$ - la ratio de indivisión ${ }^{4}$, Aristóteles aborda cómo se delimita lo que el uno

${ }^{3}$ Según expresión de Tomás de Aquino. Cfr. In Metaphysicorum, X, lect. 1, n. 1920.

4 Principalmente a lo largo de dos pasajes aborda Aristóteles la naturaleza del uno: en el libro V (1015b16-1017a6; 1017b27-1019a14) y en el libro X (1052a15- 
es en función de su opuesto, la pluralidad, con la intención de distinguir si conocemos la pluralidad a partir de la unidad, es decir, el uno a partir de lo mucho, o más bien, lo mucho a partir de la consideración de varios unos.

\section{La oposición entre lo uno y lo mucho}

La manera más radical de oponer al uno y la pluralidad, nos dice Aristóteles, es la oposición como algo indivisible y algo divisible, ya que oponerlos según este criterio nos permite abordarlos según su ratio definitoria, pues lo propio de la pluralidad es ser dividida; y lo propio de la unidad permanecer sin tal división. En efecto, para conservar la noción de casa es indispensable que no considere desvinculadas las ventanas respecto de la puerta, por ejemplo; y el techo respecto de los muebles: todo ello es casa de igual modo, por lo menos en la consideración bajo la especie casa. Al contrario, si de lo que hablo es de las muchas ventanas que hay en una casa; necesito considerar forzosamente cada ventana como diferente y dividida respecto de las demás.

Ciertamente, para hablar de la pluralidad no es necesario que algo sea ya dividido en acto o simplemente divisible en potencia, pues a lo primero claramente se le llama plural, y a lo que aún no ha sido dividido, por la facilidad de realizar en ello la división, se le llama también plural. Esto es, las partes de la casa a las que hemos aludido, por ser ya diferentes y divididas en acto, son plurales; mientras que se

1055a23) de la Metafísica. No abordaremos aquí los interesantes matices que se exponen ahi para establecer con base en dos formulaciones diferentes la naturaleza del uno, sólo recogemos, como ya se dijo, la conclusión: el uno que se convierte con el ente tiene como ratio definitoria la indivisión, mientras que el uno numérico sirve más para dividir o distinguir realidades, que para unificarlas: una casa, como designante del conjunto de entes unificados dentro de su pluralidad en base a una sola especie (uno metafísico), es diferente a decir tres casas, en donde más bien se busca distinguir (uno numérico) que unificar. A lo largo de este breve trabajo nos serviremos, como en su momento se señalará, de los apuntes y sugerencias que Tomás de Aquino hace al comentar los respectivos pasajes citados, principalmente, del los libros V y X de la Metafisica, 
dice plural el agua -al afirmar "en el océano hay mucha agua"- no porque tenga partes divididas en acto y unidas -como cuando decimos "en el bosque hay muchos árboles"- sino porque es fácilmente divisible el agua $y$ en potencia sus partes son separadas, como separadas están las gotas en la lluvia, y por lo cual se dice que hay "muchas gotas en la lluvia".

De este modo, si no son lo mismo uno y pluralidad o uno y mucho, es pertinente mostrar a qué genero de oposición se reducen, para con ello establecer cuál de ambas nociones conocemos primero. Dentro de los cuatro modos de oposición que Aristóteles distingue, ubica la oposición uno-mucho en el contexto de la contrariedad. Para que algo se oponga a otro al modo de la contradicción es necesario que a uno de ellos se le atribuya el no ente: así como se dice que "todo hombre está sentado" se opone a "ningún hombre está sentado", en donde el no ente supuesto en "ningún", respecto de "todo" se opone contradictoriamente.

Esta oposición de matiz lógico, explica su restricción ontológica dado que si el no ente necesario en la contradicción es opuesto a su extremo, en cierto sentido se le da una entidad para referirlo como opuesto. Por ello, si la contradicción fuera la manera de oponer lo uno y lo mucho, el no ente supuesto en la contradicción tendria que ser, o bien mucho, es decir, pluralidad -y estar opuesto a lo uno-; o uno, y oponerse a lo mucho. Pero, evidentemente, el no ente que es extremo en la contradicción no es ni mucho ni uno, pues no es ni una cosa ni muchas ${ }^{5}$. En la oposición segín la relación, es necesario que uno se defina por el otro, como el padre no puede ser tal si no se refiere a su hijo y viceversa ${ }^{6}$; en cambio, el uno tiene como ratio la indivisibilidad, el carácter por el cual es continuo, lo cual lo define en

\footnotetext{
${ }^{5}$ ARISTÓTELES, Metaphysica, 1054a20-26; TOMÁs DE AQUino, In Met. X, lect. 4, n. 1987: "Y que algunos no se oponen según la contradicción, es manifiesto porque ninguno de ellos se verifica acerca del no ente. En efecto, el no ente no es ni muchas cosas ni una. Convendria que una parte de la contradicción se verificara tanto del ente como del no ente."

${ }^{6}$ ARISTÓteles, Categorias, 6a36-bl4.
} 
sí mismo, y no como "aquello que no es mucho". Si se definiese así, no sólo se opondrían como relación, sino que se caería en una petición de principio.

Quizá hay más razones para pensar que uno y mucho se oponen al modo de la privación y el hábito, ya que lo uno respecto de lo mucho se podria tomar como una cierta privación: tengamos en cuenta que la ratio del uno es la indivisión, y de este modo parece oponerse a la división, pues lo indiviso o indivisible -ya sea en acto o en potenciaes lo capaz de ser dividido, y para que las partes puedan ser distinguidas en acto, deben ser unitarias en continuidad, y precisamente al ser divididas muestran que antes tenían la capacidad de tal característica. Así, un tronco de madera puede ser dividido en pedazos porque antes de la división tenia la privación de la división.

\section{El género de la oposición entre lo uno y lo mucho}

Ahora bien, si el opuesto del uno es la pluralidad, lo es porque están en el mismo género de cosas: el opuesto del uno que es el tronco de madera no es la pluralidad de las hojas del árbol, sino la multitud de los pedazos de madera en que se ha dividido el tronco. De este modo, la oposición privación-hábito tiene razón de ser cuando se comparte el mismo género, y ello sucede cuando a su vez hablamos de la oposición de contrariedad, no de simple privación-hábito; pues la raiz de ésta es la contrariedad ${ }^{7}$.

En la contrariedad, uno de los miembros siempre es privación, pero no absoluta sino en el mismo género de su contrario. Por eso debe tener una cierta naturaleza alguno de los contrarios para poder oponerse a otro en función de esa misma naturaleza, aunque uno de los contrarios posea esa naturaleza de manera precaria y el otro contrario de manera plena; en función de lo cual parece que lo indiviso se ordena a la división. De cualquier modo, no hay una

ARIstóteles, Met., X, 1054a23-26: "Puesto que hay cuatro clases de oposición, $\checkmark$ uno de estos dos términos implica la privación, se tratará de una oposición de fontrarios y no a manera de contradicción ni de relación". 
oposición entre el uno y la pluralidad -como si la privación absoluta de división se opusiese a la división- sino que el uno es indivisión del ente indiviso; esto es, indivisión de algo que ya es. Y la pluralidad es el ente ya dividido, por lo que la oposición es de contrariedad y no privativa ${ }^{8}$

\section{El conocimiento de la pluralidad a partir del uno}

Aristóteles mismo afronta una objeción tácita a lo dicho sobre la oposición al modo de la contrariedad, entre lo uno y lo mucho. En primer lugar, hay que tomar en cuenta que el uno se conoce desde su contrario, esto es, desde la pluralidad. Ya dijimos arriba que lo uno no es sino a lo que llegamos después de percibir lo mucho; así como después de percibir la puerta, ventanas y techo, podemos referimos a una casa, y si se considera desde el punto de vista de la ratio del uno y lo mucho, lo indivisible se conoce a partir de lo divisible: si no hemos percibido que el agua puede estar dividida en gotas, no podemos considerar el océano como divisible, aunque sea uno continuo.

De hecho, no es la indivisibilidad lo que captamos por cada uno de los sentidos, ya que ninguno de ellos percibe sino sólo su objeto propio; esto es, el ojo sólo percibe luz, el tacto sólo rugosidad o el oído sólo sonido, y ninguno de ellos percibe el sonido como uno, la rugosidad como una o la luz como una. Más bien, es en la integración del sentido común cuando se sabe que son varios sensibles. Incluso por la misma operatividad simultánea de la sensibilidad es que

\footnotetext{
${ }^{8}$ Según observa Tomás de Aquino, In Met., X, 4, n. 1988: "En efecto, uno de los contrarios siempre es privación, pero no privación pura; pues asi no participaria de la naturaleza del género, estando los contrarios en el mismo género. Conviene de este modo que cada uno de los contrarios sea una cierta naturaleza, aunque uno de ellos participe de al naturaleza del. género con cierto defecto, como lo negro respecto de lo blanco, como arriba ha sido dicho. de este modo ya que el uno no significa pura privación, pues no significa la indivisión misma, sino el mismo ente indiviso, es manifiesto que lo uno y la pluralidad no se oponen según la privación pura y el hábito, sino como contrarios".
} 
captamos la pluralidad, como ocurre con el número entendido como sensible común 9 .

No sólo la naturaleza de la percepción sensible aboga por la preeminencia de la pluralidad respecto a la unidad, sino que parece haber una razón ontológica: lo indivisible -y en el nombre mismo se recoge ya esto- parece privación de lo divisible; y respecto de una entidad material ya dada, individual, independiente en su singularidad o cuantificada, la indivisibilidad es posterior a la forma ya adquirida que es la singularidad. Es decir, respecto de algo múltiple, lo unitario es como su privación: la privación es la eliminación de esa individualidad que le compete por cuantificada. De este modo, la pluralidad tiene prioridad gnoseológica sobre la unidad, dada la peculiaridad de nuestro conocimiento sensible ${ }^{10}$.

A propósito de este asunto, Tomás de Aquino recuerda la dialéctica existente entre lo real y lo gnoseológicamente más importante, respecto de lo menos evidente, expuesto por Aristóteles en el primer libro de la Física. Aquello que es más importante según la naturaleza, y por ello más evidente, es posterior y menos evidente respecto de nosotros, dado que el conocimiento lo obtenemos por los sentidos, y estos comienzan su investigación de la realidad a partir de lo que nos es más evidente pero que cara a la realidad es lo menos importante. Por ejemplo, el color, la figura, el modelo e incluso el material que percibimos en una silla, es lo que primeramente adviene a nuestro intelecto, y sin embargo la esencia de silla, no la conocemos por los sentidos externos ${ }^{11}$.

\footnotetext{
${ }^{9}$ No es aqui el lugar de estudio de los requisitos para distinguir la operatividad de la sensibilidad externa de la interna; pero pueden verse con provecho los lugares típicos que en el De anima están reservados para tal efecto. Cfr. De anima, I, 418 all-424al6; 429al y ss.

${ }^{10}$ ARISTÓTEles, Met., X, 1054a26-29: "El uno se dice y se explica desde su contrario, y lo indivisible, desde lo divisible, por ser más perceptible sensorialmente la pluralidad y lo divisible que lo indivisible, de suerte que la pluralidad tiene prioridad conceptual sobre lo indivisible, a causa de la percepción sensible". il ARISTÓTELES, Física, 184a-184b4; TOMÁs DE AQUINO; In Physicorum, I, nn. 6 y 7.
} 
Supongamos que aceptamos como esencia de la silla la ordenación entre sí de un asiento individual, un soporte y un respaldo; a esta conclusión se llega posteriormente, y con mucho, luego de haber captado el color, la figura y el material de la silla. Estas características accidentales no son relevantes respecto de la naturaleza de la silla; no es más silla una Luis XV que una de jardin, o una de madera más que una de metal. De este modo, comenzamos el conocimiento de la silla a partir de lo que nos es más evidente -el conocimiento comienza por los sentidos- pero menos importante, y llegamos a lo que es más importante en la naturaleza y menos evidente para nosotros; tan no evidente es para nosotros que llegamos después de una investigación y conceptualización ${ }^{12}$.

Aún más, lo que nos es menos evidente y más importante respecto a la naturaleza, es la más actual en la cosa, y tan más actual es, que sin ello la cosa no existiria: la vinculación u ordenamiento entre sí del asiento, el soporte y el respaldo es lo más real en la silla, si no existiera esta vinculación, no habría silla. Y como es lo más real, y es lo que hace en acto la silla, entonces es lo más inteligible, porque lo actual es lo inteligible, ya que el conocimiento es la apropiación de una forma -que es acto- sin perder la propia ${ }^{13}$. Al contrario, el color, el modelo o el material, aunque es cognoscible por los sentidos externos, no constituyen la esencia en acto de la silla pues no depende de ellos el que haya silla, y por eso no es lo más real ni lo más actual.

Lo mismo podríamos decir no sólo en cuanto a lo artificial, sino en el orden de los seres naturales: si entendemos como constitutivo esencial de una estrella el que sea un cuerpo celeste que por la vinculación de sus componentes y reacciones internas irradia más

12 Tomás de Aquino, In Met., X, 4, n. 1990: "Para ver la solución de esta objeción debe considerarse que aquellas cosas que son más importantes (priora) según la naturaleza y más evidentes (magis nota), son posteriores y menos evidentes respecto de nosotros, debido a que el conocimiento de las cosas lo obtenemos por el sentido. Las cosas compuestas y no explícitas (confusa) caen primero en el sentido, como se dice en el primer libro de la Fisica".

${ }^{13}$ ARISTÓTEles, De anima, II, 424al 8 y ss.; Ш, 429a18-431b21. 
energía de la que recibe, llegamos a ello no por los sentidos externos. Es más, primero, llegamos -como desde el hombre de las cavernas pudo haberse establecido- a que una estrella es algo que brilla y parpadea en la oscuridad de la noche. Esto es más evidente para nosotros, pero cara a la realidad es lo menos importante, pues también los planetas brillan y no son estrellas porque brillen. Y lo más esencial, lo más acto de la estrella -el hecho de que las reacciones hagan que irradie más energia de la que recibe- se conoce después, cuando se llega a lo que nos era menos evidente pero más real, actual, importante para la naturaleza.

En el mismo pasaje de la Física se expone cómo primero advienen a nuestros sentidos las realidades no explicitas o compuestas. Como cuando vemos que se acerca un bulto y lo percibimos como tal o como un manchón en la neblina; esto es, el conocimiento confuso, no explicitado. Sólo se trata de una realidad de la cual no se sabe qué detalles posea, y que no se explicitan en un primer momento. Posteriormente, se van conociendo más detalles de esa realidad, y se pasa de concebir un simple bulto a ver que es un hombre y luego que es Sócrates ${ }^{14}$.

Asi, viene a nuestro conocimiento primero lo compuesto y luego lo simple; primero lo confuso y luego lo explícito. Y sin embargo, lo simple ya existe en acto en la realidad que nosotros conocemos como compuesta. La singularidad de Sócrates ya existe cuando nosotros de manera no explícita sólo percibimos un bulto que se acerca. De este modo, lo simple es anterior en la realidad a lo compuesto, y lo compuesto es anterior en el conocimiento a lo simple. Y, con todo, lo simple es más cognoscible por más real y actual que lo compuesto, lo cual está en potencia, no en acto. Incluso, por ejemplo, hablando del conocimiento de los principios, éste se da a partir de las negaciones de lo que es posterior en la naturaleza, pero primero en el conocimiento.

${ }^{14}$ ARIstóteles, Fisica, 184a-184b4; Tomás de Aquino; In Phys., I, nn. 6 y 7. 
Como cuando decimos que conocemos el principio de no contradicción -que es primero en la naturaleza, y posterior en el conocimiento- después de negar que dos cosas pueden ser al mismo tiempo y bajo las mismas circunstancias; primero conocemos esas dos cosas y las circunstancias. Y de negar su sumultaneidad en el ser, la cual percibimos de manera primera, se explica el primer principio, al cual llegamos después ${ }^{15}$.

O como ocurre con el punto, al definirlo como lo que no tiene partes, pues primero conocemos las partes. Asi, el uno se define a partir de la pluralidad como privación de la divisibilidad que percibimos por los sentidos. Por eso el uno se conoce a partir de su contrario, pues es más sensible la multiplicidad que la unidad. Y, según su consideración, es anterior la multiplicidad a la unidad, pero no en el orden del ser, sino según la sensibilidad, punto de partida de nuestro conocimiento ${ }^{16}$.

El fundamento del conocimiento de la unidad a partir de la pluralidad, depende, asi, de que ambos se opongan como contrarios. Sin embargo, respecto a dicha contrariedad, Aquino establece una doble duda al comentar este pasaje: por un lado, parece que lo uno es lo que constituye la multiplicidad, de modo que el bosque como

15 TOMÁs DE AQUUNo, In Met., X, 4, n. 1990: "Y por ello es que las cosas compuestas vienen primero a nuestro conocimiento. Pero las más simples que son anteriores y más evidentes según la naturaleza, vienen a nuestro conocimiento posteriormente. Por ello es que los primeros principios de las cosas no las definimos sino por las negaciones de las cosas posteriores, así como decimos que el punto es de lo cual no hay parte; y conocemos a Dios por las negaciones, en cuanto que decimos que Dios es incorpóreo, inmóvil, infinito".

${ }^{16}$ Tomás DE AQuino, In Met., X, 4, n. 1991: "Y asi de este modo aunque 10 uno sea anterior según la naturaleza a la multiplicidad, sin embargo según nuestro conocimiento se define y se nombra a partir de la privación de la división. A causa de esto dice el Filósofo que "el mismo uno se dice", esto es se nombra "y se muestra", esto es, se conoce, a partir de su contrario; como lo indivisible a partir de lo divisible: a causa de que la multiplicidad es más sensible que lo uno, y lo indivisible más que lo divisible. Por lo cual la multiplicidad es anterior según la razón a lo indivisible, pero no según el orden de la naturaleza, sino según el sentido que es principio de nuestro conocimiento". 
unidad no es otra cosa que el conjunto de unos que son los troncos. En la contrariedad, en cambio, un extremo de ella no constituye al otro extremo, antes bien lo aniquila. Con la cual se sigue la inconsencuencia de que la realidad a partir de la cual conocemos la pluralidad debía anularla, más que permitimos conocerla.

\section{La prioridad ontológica del uno frente a la multiplicidad}

En la contrariedad, apuntábamos arriba, se da la oposición en el mismo género; esto es, en virtud de una forma común, o un acto que relacione los extremos. Y de ambos extremos se dice la forma; esto no quiere decir que uno de los extremos sólo sea parte de lo que tiene forma: como si dijésemos que la unidad fuese una parte de forma, y la pluralidad la forma completa.

Por ejemplo, si se toma el alma separada ya del cuerpo, aquélla tiene ya el acto o la forma, y se opone al cuerpo como lo animado a lo inanimado, porque ambos poseen la misma forma, y de ambos se dice. Y de este modo, el cuerpo que antes estaba vivo y le competía la animación, se opone al alma en el mismo género, pues ésta posee el acto que hace la animación, y el cuerpo, una vez separada aquélla de éste, ya no tiene la animación.

Pero si se le considera como algo que está en potencia de llegar a su tamaño ideal o al peso que le compete por naturaleza, entonces no se opone a animado, sino que es parte del mismo animado. Es decir, al animal le compete incluir dentró de él una materialidad que está en constante cambio, y a la cual "le falta" aquello a lo que tiende, pues de lo contrario no habria tal tendencia. Y asi un ser en crecimiento, se opone a su mayor tamaño como lo imperfecto a lo perfecto.

Otro caso: lo doble se opone a lo triple según la misma especie de número y de ambos se predica la misma forma determinada; sin embargo, desde el punto de vista de que lo doble puede admitir incremento -porque no es todo el número posible-, se puede llegar a lo triple, al añadir la unidad al doble. Asi, lo doble, como número, tiene 
tal forma, e igualmente como lo triple; pero si se toma como número capaz de recibir otra unidad, entonces es parte de lo triple.

De igual manera, si el uno se opone a la multiplicidad, y tomamos en cuenta que ambas realidades comparten una forma determinada, entonces es como considerar al uno perfecto en sí mismo e independiente de cualquier otra referencia; y frente a la multiplicidad, a su vez, ésta posee una forma que la hace independiente y opuesta al uno.

Pero si el uno, en cambio, se considera como privado de división, esto es, incompleto, por lo menos en ese aspecto -ya que está ordenado a algo que no posee aún-, no se puede oponer a la pluralidad, sino que más bien es parte de ella. Y de este modo, si la supuesta oposición uno-multiplicidad es como la existente entre privación-hábito, uno y pluralidad no son contrarios ${ }^{17}$.

En un segundo argumento, Tomás de Aquino advierte la aparente conculcación de la anterioridad de lo plural respecto del uno. Y es que

17 TomÁs DE AQUino, In Met. X, 4, nn. 1992-1994: "Pero contra aquellas cosas que determina aquí el Filósofo, surge una doble duda. La primera acerca de que lo uno y lo mucho se oponen como contrarios. En efecto, esto parece imposible porque lo uno constituye la multiplicidad; y uno de los contrarios no constituye al otro, sino que más bien lo destruye. Debe entenderse de este modo, que difiriendo los contrarios según la forma, como se dice abajo, cuando decimos que algunas cosas son contrarias, debe considerarse cada una de ellas en cuanto que tiene forma, y no en cuanto es parte de lo que tiene forma. En efecto, el cuerpo sin alma, en cuanto que se toma como lo que tiene forma, se opone al animal como lo inanimado a lo animado. Pero en cuanto que se toma como algo casi no perfecto y formado, no se opone a lo animal, sino que es parte material de él mismo. Y así también lo vemos en los números. Pues lo binario en cuanto que es cierto todo, que tiene una especie y forma determinada, es diferente en éspecie respecto de lo triple; pero si se considera al margen de que sea perfecto por la forma, es parte de lo triple.

De igual manera, el mismo uno, en cuanto que se considera como perfecto en si mismo y poseedor de cierta especie, se opone a la multitud, porque lo que es uno no es muchas cosas ni viceversa. Pero en cuanto que se considera como no completo según la especie y la forma, de este modo, no se opone a la multiplicidad, sino que es parte de ella". 
si la multiplicidad no es sino agregado de unidades, hay circularidad en el conocimiento de la unidad: por un lado la pluralidad es un conjunto de unidades; y por otro, es necesaria la pluralidad para conocer la unidad.

Si esta circularidad se da, sería como afirmar que si se define el uno con base en la pluralidad y ésta con base en la unidad, lo menos evidente se conocería a partir de lo más evidente, y lo más evidente se conocería a partir de lo menos evidente; como si la esencia de la silla se conociera a partir de su color, y como si el color se conociera a partir de la esencia de la silla. O la esencia de la estrella a partir de que brilla, o el brillo a partir de la esencia de la estrella ${ }^{18}$.

Respecto de esta aporia, según Tomás de Aquino, no hay contradicción en que algo sea anterior y posterior respecto de lo mismo, según una diferente consideración racional (ratione), aunque en si mismo sean diferentes realidades ${ }^{19}$. Asi, la multiplicidad que es el bosque puede verse ya como multiplicidad o ya como división, esto es, se puede considerar primero la individualidad de los troncos (división), lo cual da origen a la multiplicidad, o bien primero puede considerarse la multiplicidad, a partir de la cual posteriormente se consideran los individuos que la integran.

\footnotetext{
${ }^{18}$ Ciertamente muchas de las características esenciales de las estrellas se conocen después de descomponer el espectro de la luz resultante de su brillo. Pero aquí hablamos más bien del mero hecho de brillar, el cual no me da la esencia de la estrella, aunque se pueda descomponer después su espectro y conocer su composición particular.

${ }^{19}$ TOMÁs DE Aquino, In Met. X, 4, n. 1995: "La segunda duda nace acerca de que la multiplicidad es anterior por la razón (ratione) al uno. En efecto, siendo lo uno propio de la naturaleza (de ratione) de la multiplicidad, debido a que la multiplicidad no es otra cosa que la agregación de unidades, si el mismo uno es posterior por la razón a la multiplicidad, se sigue que en la naturaleza del uno y de la multiplicidad, hay cierto círculo; de modo que sea necesario para entender (intelligi) la multiplicidad, la unidad; y viceversa. Pero no ocurre el círculo en las definiciones de las cosas, porque sería lo mismo por lo mismo más y menos evidente, lo cual es imposible".
} 
Veamos el asunto desde la perspectiva uno-mucho: lo mucho -según la ratio arriba señalada- es división, pluralidad, por lo cual, desde la consideración de la división, es anterior al uno; pero desde el punto de vista de la multiplicidad, es posterior al uno, porque multiplicidad es un conjunto de unidades.

Ahora bien, parece no haber conflicto ni circularidad, porque la división supuesta en la naturaleza del uno que se convierte con el ente, no es la división de la cantidad continua; precisamente la división de la cantidad continua está presupuesta pero en el uno que no se convierte con el ente, es decir el numérico, cuya ratio consiste precisamente en la división, en crear distinción cuantitativa ${ }^{20}$. La división del ente que se convierte con el ente, más que dividir crea contrariedad, "distancia" entre los entes que se dicen "divididos" porque uno no es el otro. Pero el que un sujeto no sea otro no está determinado en primera instancia porque la cantidad de uno esté separado de la cantidad del otro.

La alteridad no radica primeramente en los límites de cada una de las cantidades, sino que la alteridad más bien está determinada por la oposición de contrariedad. Y asi no hay circularidad, ya que en la multiplicidad del bosque está supuesto el conjunto de unidades que son los árboles, pero no como diferentes primeramente por los límites de su cantidad, sino primeramente como contrarios: un tronco no es el otro, y ello es indispensable para que existan varios -lo cual constituye al bosque-. Y conocemos primero la multiplicidad, y luego la singularidad; pero el conjunto de unidades supuesto en la multiplicidad no es un conjunto de singularidades, sino de distinciones contrarias que fundamenta el que la pluralidad sea un grupo de realidades diferentes, más que por los limites cuantitativos, por la contrariedad",21.

${ }^{20}$ Sobre el uno numérico, que no se convierte con el ente, cfr. ARISTÓTELES, Met., IV, 1004a2-1005a18; y V, 1016b3-1017a6.

${ }^{21}$ Cfr. TOMÁs DE AQuino, In Met. X, 4, n. 1996-1997: "Debe decirse, que nada prohibe que algo sea anterior y posterior a lo mismo según la razón, pero diferente considerado en ello mísmo. En efecto, en la multiplicidad puede considerarse tanto 
$Y$ esto es lo medular a destacar en este breve acercamiento que hemos intentado: si la unidad supuesta en la pluralidad no es singularidad, entonces es posible que a partir de la pluralidad conozcamos la unidad, sin argumentar circularmente. Esto es, primero conocemos en el bosque, que hay entes; luego, conocemos la división, es decir, que unos entes no son otros, pero por contrariedad entre ellos, no por los simples límites cuantitativos -esto es, no por simple singularidad-. Después conocemos el uno que es privación de esa división, y que -ahora sí- distingue los diferentes individuos como tales; y al final, la pluralidad, que es el conjunto de dichos individuos.

¿Por qué si están divididos los árboles del bosque -es decir, si son diferentes, o contrarios-, no son pluralidad sino hasta después de considerarlos como individuos por el uno que priva tal división? Porque aunque por la contrariedad están divididos los árboles no tienen ratio de muchos sino después de que a cualquiera de ellos se les atribuye que sean uno, lo cual implica que como cualquiera de ellos es uno, pueda haber pluralidad, entendida ésta como conjunto de esos unos; y entonces sí, el bosque es una pluralidad de árboles. Si la pluralidad dependiera de un conjunto de unos y los entendiéramos como el uno que convierte con el ente, la pluralidad dependería en su ser de la indivisión, lo cual sí sería contradictorio. Más bien, en su seno la pluralidad depende del uno numérico; el cual, en cambio, precisamente tiene como ratio la división ${ }^{22}$.

que es multiplicidad como la división misma. De este modo, por la razón de la división, es anterior al uno según la razón. En efecto, (nam) lo uno es lo que no se divide. Pero en cuanto es multiplicidad, es posterior al uno según la razón, pues se dice multiplicidad, la agregación de unidades. Pero la división que se presupone respecto de la naturaleza del uno, en cuanto que se convierte con el ente, no es la división de la cantidad continua, la cual se sobreentiende en el uno que es principio del número; sino que es la división que causa la contradicción en cuanto

que este ente y aquél se dicen divididos, debido a que éste no es aquél".
$2_{2}$ Cfr. TOMÁs DE AQUINO, In Met., X, 4, n.1998. "Por lo tanto, así, primero viene a nuestro intelecto el ente, $y$ después la división, $y$ posteriormente el uno que priva la división, y al último la multiplicidad que se constituye de unidades. En efecto, aunque aquellas cosas que son divididas sean muchas, no tienen, sin embargo, naturaleza de muchos, sino después de que a este 0 aquél se les atribuye que sean uno. Sin embargo nada prohibiria decir que la naturaleza de la multiplicidad 
La realidad de la unidad supone, como hemos adelantado ya al abordar su relación con la pluralidad, su contrario. Pero hay tres realidades que a su vez siguen al uno como partes ${ }^{23}$ : la identidad, la semejanza y la igualdad ${ }^{24}$. Y asimismo, como partes de su contrario, la pluralidad-, le siguen la alteridad, la desemejanza y la desigualdad $^{25}$. En donde idéntico es aquello que es uno en la sustancia. Respecto de esto Aristóteles asume varios matices: hay identidad, porque hay unidad en la sustancia, cuando decimos que Sócrates es idéntico con lo blanco de su piel, porque es la misma sustancia la de Sócrates y en la que se encuentra su blancura.

$\mathrm{O}$ bien, yo con relación a mí mismo soy idéntico por el enunciado; esto es, soy en mi materialidad una sola sustancia, pues todas las partes de mi cuerpo pertenecen a la misma materia, de la cual parte mi individuación como sujeto. Y desde el punto de vista de la especie, también hay identidad, pues la especie se toma como naturaleza del sujeto, y por eso, todo lo que se diga de la especie, al definir en el enunciado el sujeto que soy yo, se dice de una sola sustancia que es constituida por mí. Y de igual modo, hay identidad, porque comparten la misma especie, entre las figuras geométricas o entre dos líneas ${ }^{26}$.

En cambio, lo semejante es lo uno en la cualidad, esto es, como una figura geométrica mayor es semejante a una pequeña, y la unidad entre ellas se toma según la proporción; y también hay semejanza entre rectas desiguales, porque comparten al misma cualidad. Son

depende del uno, en cuanto que es medida por el uno, lo cual ya pertenece a la naturaleza del número".

${ }^{23}$ En este sentido hay que tomar en cuenta la distinción que el mismo Aristóteles establece, entre otros pasajes, en el libro V de la Metafisica; en donde muestra la posibilidad de asumir partes no cuantitativas. Es así como entiende las partes del uno. Cfr. Met., V, 1023a26-1024a28.

24 En efecto, idéntico es lo uno en la sustancia, semejante lo uno en cualidad, e igual lo uno en la cantidad. ARISTóteles, Met. V, 1017b27-1018b8.

${ }^{23}$ Las cosas diversas son de las cuales no hay una sola sustancia, y semejantes las cuales no tienen una sola cualidad, y desiguales las que no tienen una sola cantidad. Cfr. ARIstóteles, Met., X, $1054 a 29$ y ss.

${ }^{26}$ ARIstóteles, Met., X, 1054a32-b3. 
también semejantes las cosas cuya afección es idéntica y especificamente una, como la blancura intensa y la blancura tenue. $\mathrm{O}$ como mayor y menor se dicen semejantes porque su especie es una ${ }^{27}$. $Y$ lo igual es lo uno en la cantidad, como ocurre entre dos caballos y dos casas; y no importa la comunidad de especie o sustancia o forma.

Todas estas variantes de la dialéctica unidad-pluralidad involucran elementos adicionales que no analizaremos aqui, pues rebasan el objeto de este pequeño acercamiento. Ciertamente, la identidad, la semejanza y la igualdad, junto con sus opuestos, lo desemejante y lo diferente originan nuevos matices que complementan la prioridad ontológica de la unidad frente a la pluralidad. Hemos querido advertir cómo en Aristóteles se muestra que la oposición por contrariedad rige la realidad; pero para poder asumirla debe ponerse en claro antes la prioridad gnoseológica de la pluralidad frente a la unidad, y la paradójica prioridad ontológica de la unidad frente a la pluralidad, como hemos apuntado ya ${ }^{28}$.

\section{${ }_{28}^{27}$ ARISTóteles, Met., X, 1054b3-13.}

28 El tema de la pluralidad y la unidad, no sólo por la dialéctica existente entre ellos, sino por su papel en la investigación de la realidad natural, ha sido abordado con provecho, entre otros, por REALE, G., "Struttura paradigmatica e dimensione epocale della metafisica di Aristotele: "henologia" e "ontologia" a confronto", en AAVV, Perchè la Metafisica, Vita e Pensiero: Milano 1994, pp.37-58. LowE, F., "Aristotle on Being and the One", en Archive für Geschichte der Philosophie, 59 (1977), pp.213-227. ElDERs, L., Aristotle's Theory of the One, Assen, 1961. RungGaldiER, E., "Einheit und Identität als "Begriffe" in der Metaphysik des Aristoteles", Theologie und Philosophie, 64 (1989), pp.557-559. JEANnOT, "Plato and Aristotle on Being and Unity", The New Scolasticism, 60 (1986), pp.404-426. GILBERT, P., "La métaphysique, l'acte et l'un", Gregorianum, 73, 2 (1992), pp.291-315. Fragata, J., "O problema do Uno e do Multiplo", Revista portuguesa de filosofia, 1980 (36), pp.227-248. 
Copyright of Tópicos. Revista de Filosofía is the property of Universidad Panamericana and its content may not be copied or emailed to multiple sites or posted to a listserv without the copyright holder's express written permission. However, users may print, download, or email articles for individual use. 